\title{
LOS MEGALITOS DE TÉRMINO. CRÓNICA DEL VALOR TERRITORIAL DE LOS MONUMENTOS MEGALÍTICOS A PARTIR DE LAS FUENTES ESCRITAS
}

\author{
MEGALITHS AS LAND-MARKS. CHRONICLE OF THE TERRITORIAL ROLE \\ OF THE MEGALITHIC MONUMENTS THROUGH WRITTEN SOURCES
}

\author{
MARCOS MARTINÓN-TORRES (*)
}

\begin{abstract}
"A megalith in an urban environment does not work, it has no aura... By contrast a megalith in a rural setting, in fields or woods, always has a distinctive atmosphere and character about it."

(Christopher Tilley, 1993: 163-164)
\end{abstract}

\section{RESUMEN}

A lo largo de la Historia, los monumentos megalíticos han desempeñado, entre otras, una función espacial, como marcos de territorio. Para este artículo se recogen y analizan las referencias escritas a megalitos gallegos funcionando como marcadores o identificadores espaciales, entre los siglos VI y XIX d.C.A partir de este registro de fuentes se reconstruye la evolución de este papel social-territorial de los monumentos en las distintas épocas. Se plantea un modelo interpretativo para este fenómeno, y se valora la revisión de fuentes escritas como metodología para la prospección arqueológica y para los estudios de emplazamiento de megalitos.

\begin{abstract}
Megalithic monuments have played different roles throughout History. One of them has a spatial function, i.e. as landmarks. The aim of this paper has been to collect and analyse every written reference concerning Galician megaliths operating as landmarks between the 6th and 19th centuries $A D$. On this basis, the evolution of this social-territorial function of the monuments through time is reconstructed, and an interpretative hypothesis for this phenomenon is proposed. Finally, the importance of reviewing
\end{abstract}

(*) Dpto. de Historia I. Universidad de Santiago de Compostela. Pza. da Universidade, 1. 15703 Santiago de Compostela. Correo electrónico: marcosmartinon@mixmail.com

El artículo fue remitido en su versión final el 30-XII-2000. written sources as a methodology for archaeological survey and for studies of the topographic settings of monuments is emphasised.

Palabras clave: Megalitismo. Historiografía. Fuentes documentales. Galicia. Arqueología del Paisaje. Territorialidad. Prospección arqueológica. Reutilización de los monumentos.

Key words: Megaliths. Historiography. Documentary sources. NW Iberia. Landscape Archaeology. Territoriality. Archaeological survey. Reuse of monuments.

\section{INTRODUCCIÓN}

Dentro de un proyecto más amplio de revisión documental e historiográfica del Megalitismo gallego, hemos definido tres valores o potenciales de los monumentos megalíticos. Estos valores se han apreciado con intensidad variable en las distintas épocas históricas, en función de los diversos contextos socioculturales (Martinón-Torres y Rodríguez Casal, 2000; Martinón-Torres, e.p.b):

a) un valor social-territorial, por el empleo de los megalitos como marcadores territoriales;

b) un valor simbólico-mítico, por el atractivo idealizado que ejercen sobre los buscadores de tesoros, así como por su inclusión en variables formulaciones míticas; y

c) un papel histórico-arqueológico, que apare-

T. P., 58, n. ${ }^{\circ} 1,2001$ 
cerá cuando surja el interés por referirse a los megalitos en sí mismos, como vestigios de un pasado remoto acerca del cual pueden informar.

Este trabajo se centrará sobre el primero de estos aspectos: la funcionalidad del monumento megalítico como marco de territorio. A partir del vaciado sistemático de fuentes escritas medievales, modernas y contemporáneas, trataremos de reconstruir la evolución de este empleo práctico de los megalitos y de sugerir un modelo explicativo que nos ayude a comprender este fenómeno. Asimismo, tomaremos esos datos como base para plantear líneas de trabajo e interpretación arqueológicos que apenas se cultivan y que consideramos, no obstante, de gran interés. Hablaremos, por tanto, de megalitos de término, parafraseando a Jesús Ferro Couselo (1952). Él fue el primero en abordar sistemáticamente el estudio de restos prehistóricos gallegos a través de las fuentes históricas, y su trabajo fue durante mucho tiempo debatido e infravalorado (Rodríguez González, 1996). Partimos ahora de su estela esperando llegar a fines distintos, pero sin dejar de reconocer su legado.

\section{LOS MEGALITOS DE TÉRMINO EN LA PREHISTORIA}

En las últimas décadas, la mayor parte de las interpretaciones en Prehistoria y Arqueología insisten en los aspectos funcionales del objeto estudiado. Para el caso concreto del Megalitismo, esos modelos interpretativos de carácter "utilitarista" se han orientado en diversas direcciones, por ejemplo para acentuar el papel simbólico y religioso de los monumentos o para subrayar una función más social, vinculada a la demarcación y organización del espacio en que un grupo existe (Criado, 1991a). Más recientemente, las Arqueologías Postprocesuales han criticado -con mayor o menor firmeza- las anteriores perspectivas, que partían del funcionalismo y de la New Archaeology, para dirigir sus hipótesis hacia otros postulados. Sin embargo, en un sentido amplio, parece innegable que no se ha abandonado el eterno para qué sirve formulado ante los restos arqueológicos.

La dimensión funcional que más nos interesa aquí es la que se refiere a la utilidad del monumento megalítico como marcador de territorio. Precisamente por su monumentalidad patente en el paisaje, se ha indicado repetidas veces que los megalitos habrían ejercido, en su época de construcción, un papel activo, marcando el espacio en que eran ubicados. Así, ya a principios del siglo XVII, en Inglaterra, William Camdem había concebido la posibilidad de que Silbury Hill se hubiese construido con alguna intención demarcadora (Trigger, 1992: 75). Para el ámbito gallego, sobre el que se centrará nuestro análisis, el valor territorial de los túmulos había sido sugerido por Manuel Murguía (1888: $85 \mathrm{ss}$.) a finales del siglo pasado. En una línea similar, otros autores irían señalando la vinculación entre monumentos y remotas demarcaciones o caminos, para llegar a plantear, incluso, que la función de indicadores podía anteponerse a la ritual o funeraria (Barros Sivelo, 1875; Díez Sanjurjo, 1904; Amor Meilán, 1918: 34 y 45; Castillo, 1927; Maciñeira, 1942: 23, 1947: 32 ss.).

De todas formas, esta posibilidad no adquirió solidez hasta que Fleming y Renfrew la expusieron rigurosamente. Como es sabido, el primero de ellos llamó a los megalitos "tumbas para los vivos", porque'estructuraban el paisaje y consolidaban las jerarquías de las sociedades campesinas (Fleming, 1973). El segundo, en cambio, habló de sociedades segmentarias, sin redes piramidales de poder, pero también defendió de manera sistemática la función de estas construcciones como marcos de territorio (Renfrew, 1975, 1976, 1983). Estas propuestas fueron ampliamente aceptadas en el tiempo de auge del capitalismo industrial, que facilitaba la comprensión de la sociedad como una "unidad globalizadora que funciona en aras de un equilibrio maximizador" (Criado, 1989a: 77).

La estela del funcionalismo sigue viva aunque, en los últimos quince años, las transformaciones socioculturales y el avance de la investigación han llevado a replantear los factores que condicionan el emplazamiento de los megalitos, así como sus funciones sociales. El relativismo cultural nos ha obligado, cuando menos, a ser prudentes. De este modo, el valor territorial de las construcciones megalíticas se presenta como uno más entre otros factores o condicionantes de su ubicación, ya sean de carácter geológico, topográfico, económico y/o cultural (por ejemplo, Rodríguez Casal, 1990: 4049; Vaquero, 1991-92; Criado y Vaquero, 1993; Villoch, 1995). La función territorial se expresa de una forma más vaga, diciendo que el megalito "de alguna manera presidiría un territorio de explotación" (Rojo, 1994: 85), explotación que podría ser, por ejemplo, un pasto de verano (Díez et alii, 1995). Asimismo, ha tendido a incidirse más en su carácter de marcadores (y/o señales de apropiación) de

T. P., 58, n. $^{\circ} 1,2001$ 
"lugares de paso o tránsito", en lugar de hablar de "marcadores territoriales fijos", término que transmitía una idea de inmovilidad (Galán y Martín, 1991-92; Criado y Vaquero, 1993; Teira 1994: 98; Ramil, 1997; Criado y Villoch, 1998; Andrés, 1999; Eguileta, 1997, 1999: 237-268). Otras aproximaciones plantean que los monumentos sólo habrían funcionado como verdaderos referentes territoriales en un primer momento, hasta que las poblaciones se fueron sedentarizando (Sherratt, 1990; Delibes et alii, 1997); o que lo que señalaban estos megalitos eran, en realidad, accidentes naturales con valor simbólico (Tilley, 1993, 1994, 1996). Sublimándose cada vez más la preponderancia de la función social-territorial de los monumentos, algunos investigadores han pasado de considerar los megalitos como land-marks a estudiarlos, más bien, como time-marks (Beguiristáin y Vélaz, 1999; véase también Shee Twohig, 1997).

No dejan de surgir múltiples corrientes de análisis, que no hacen sino enriquecer el panorama interpretativo. Sin embargo, no pretendemos ahora enmarcarnos en ninguna de ellas. Únicamente nos limitaremos a recordar algo que, por encima de cualquier perspectiva particular, nos resulta incontrovertible: los megalitos, en la época en que se erigieron, tuvieron un valor territorial. Consideremos ese papel en un sentido más restringido o más amplio. Hablemos de megalitos vinculados a territorios, propiedades, espacios de pasto o explotación, caminos o lugares de tránsito. En cualquier caso, está claro que los monumentos megalíticos, como elementos sólidos y visibles, señalaban el territorio, marcaban el espacio en que se ubicaban. Tener presente esta idea de un modo genérico es suficiente para los fines que nos proponemos con este trabajo. Más adelante la retomaremos.

\section{LOS MEGALITOS DE TÉRMINO EN ÉPOCAS HISTÓRICAS: GALICIA}

Dejando atrás la Prehistoria, damos ahora un salto en el tiempo para llegar hasta la Edad Media, pero sin olvidar nuestro objeto de análisis: el megalito de término. Asimismo, nos centramos en el Noroeste peninsular como zona de estudio. En esta época y en este espacio encontraremos, de nuevo, monumentos megalíticos funcionando como marcos de territorio.

Fue el Padre Sarmiento, gran ilustrado dieciochesco y escrupuloso observador de la realidad gallega, el primero en apreciar la utilización de túmulos como mojones que servían de límites para diversas propiedades territoriales. Consultando documentos medievales, se ve sorprendido por la frecuente aparición de manmulas en "término por donde pasa apeo ó demarcación en los privilegios" (Sarmiento, 1850: 73). Esta idea la retomará Manuel Murguía, a finales del siglo XIX, para recoger ya ejemplos concretos, tomados de la documentación que había publicado el Padre Flórez en la España Sagrada (cfr. Martinón-Torres, 2000b, e.p.b).

Sucesivamente, otros historiadores señalarán este hecho (por ejemplo Amor Meilán, 1918: 4647), hasta que a mediados de este siglo encontremos un primer trabajo que recoge las referencias documentales a megalitos y busca después estos monumentos en el campo (Ferro Couselo, 1952). Continuando su labor, en fechas más recientes se acometió la labor de vaciar algunas compilaciones de documentos, registrando estas citas a megalitos de término' (Filgueira Valverde y García Alén, 1977; Criado y Grajal, 1981; Pena Graña, 1991; Carneiro, 1995).

De este modo se empezó a constatar con claridad el valor territorial de los monumentos megalíticos en épocas históricas, la costumbre de emplearlos como demarcadores espaciales. Sin embargo, todos los estudios se circunscribían a espacios muy reducidos, y en ninguno de ellos se analizaba la evolución de este uso ni se sugerían explicaciones al fenómeno. Por eso consideramos conveniente sumergirnos en la documentación antigua de un modo más exhaustivo y global, para estudiar ese papel territorial del megalito en la Historia sin dejar de vincularlo con el uso que tuvieron en la Prehistoria, y empezar a sugerir criterios de interpretación.

\section{LAS FUENTES ESCRITAS GALLEGAS: LOS DATOS}

\subsection{Fuentes escritas y metodología}

El primer paso necesario fue la elaboración de una lista de topónimos susceptibles de referirse a megalitos, dada la enorme variedad de términos empleados en Galicia -como en otros lugares-para aludir a estos restos arqueológicos. Así pretendíamos evitar que se pasase por alto alguna referencia. Tomando como base inicial los trabajos de Monteagudo (1954) y de Filgueira Valverde y GarcíaAlén 
(1977), confeccionamos una tabla con 134 topónimos. Sobre esa tabla llevamos a cabo una selección, para desechar aquellas denominaciones demasiado vagas o polisémicas (casa, forno, montiño, tumba...) y quedarnos sólo con aquellas de significado más unívoco. Finalmente tuvimos una lista de los 65 topónimos con más posibilidades reales de referirse a monumentos megalíticos, y procedimos a buscar esos términos en las fuentes escritas en que podían aparecer los monumentos citados como mojones (1).

Las fuentes objeto de nuestro análisis se agrupan en tres categorías:

a) Tumbos y colecciones diplomáticas (siglos VI-XVI): En estas recopilaciones de documentos se recogen cartas de donación, testamentos, compraventas, contratos forales, confirmaciones de privilegios, etc., en los que se alude a terrenos cuyos límites se pretenden precisar al máximo. Para este fin, los márgenes de los mismos aparecerán definidos mediante marcos y, muchas veces, los túmulos megalíticos, menhires, petroglifos, castros... cumplen esa función desde tiempos remotos. Se encuentran, además, numerosos montes, valles, parroquias, granjas y otros espacios concretos que, por la presencia de un monumento antiguo, pasan a recibir su denominación. Nuestra revisión se realizó sobre una treintena de colecciones diplomáticas, precedentes en su mayoría de instituciones religiosas dispersas por todo el territorio gallego.

b) Catastro del Marqués de la Ensenada (siglo XVIII): En 1749, la Corona Española encarga al Ministro Zenón de Somodevila, Marqués de la Ensenada, la elaboración de un censo de propiedades. Su intención era reformar el sistema tributario y establecer una "única contribución" en función de la riqueza poseída. Dada su finalidad fiscal, toda propiedad, personal o institucional, debía ser declarada al Catastro, compuesto por cinco secciones: Interrogatorio General, Personal de Clérigos, Personal de Laicos, Real de Clérigos y Real de Laicos.

El Interrogatorio General consta de cuarenta preguntas, de las cuales nos interesará especialmente la número 3: "Qué territorio ocupa el término,

(1) Las múltiples denominaciones de los megalitos parten en unos casos de la forma de las construcciones (mámoa, forno, casota...). En otros, del folklore asociado a ellas (Casa dos Mouros). Finalmente, a veces el topónimo alude ya a la función que ejercen en el paisaje (anta, teriñuelo, marcón...). Para leer este artículo bastará tener presente que los términos mámoa, medoña, medorra, anta y arca, con sus derivados, son los más comúnmente empleados en Galicia para referirse a los túmulos y dólmenes. quanto de Levante a Poniente, y de Norte a Sur: y quanto de circunferencia, por horas y leguas: qué linderos, ó confrontaciones; y qué figura tiene, poniéndola al margen".

A modo de sondeo, seleccionamos como espacio la provincia antigua de Betanzos y revisamos las respuestas a esa pregunta en las 266 feligresías que la conformaban. De este modo comprobamos cómo, en muchos casos, los marcos utilizados como "confrontaciones" son monumentos megalíticos.

c) Diccionarios de topónimos (siglo XIX): Para buscar referencias a megalitos como identificadores de territorio en el siglo XIX acudimos a la toponimia y a la microtoponimia. Para ello partimos de los trabajos de José de Villarroel (1810) y Pascual Madoz (1845-50), y recogimos todos los nombres de lugar que aludiesen a posibles monumentos megalíticos.

A continuación sintetizaremos los resultados de nuéstro registro de fuentes, para presentar después las hipótesis y perspectivas que puede plantear para trabajos venideros (Fig. 1).

\subsection{Resultados (2)}

Comencemos por el principio. En el año 569, según las noticias que tenemos, se habría reunido en Lugo un concilio a instancias del obispo Teodomiro. En él se crean nuevas diócesis en la provincia eclesiástica de Galicia, a partir de la división de la antigua metrópolis en dos circunscripciones: Braga y Lugo. Las decisiones tomadas en los concilios celebrados en Lugo y, posteriormente, en Braga (572) se debieron hacer públicas, según Da Costa (1965: 16-18) entre 572 y 585 , y constituyen lo que hoy conocemos como Parroquial Suevo o División de Teodomiro.

En esta documentación se recoge la relación de parroquias que integran cada una de las diócesis, y se establecen sus límites territoriales. Para llevar a cabo esta delimitación se recurre a caminos antiguos, montes, fuentes... y también a restos arqueológicos que actúan como marcos de territorio. En particular, cuando se expone el límite de un conda-

(2) Razones de espacio nos impiden incluir un anexo con todas las bases de datos resultantes de nuestro registro de fuentes, por lo que sólo se presentará un breve resumen. No obstante, una parte sustancial de las mismas -aunque antes de ser ampliada y corregida para este trabajo- puede consultarse en Martinón-Torres, e.p.b.

T. P., 58, n. $^{\circ} 1,2001$ 


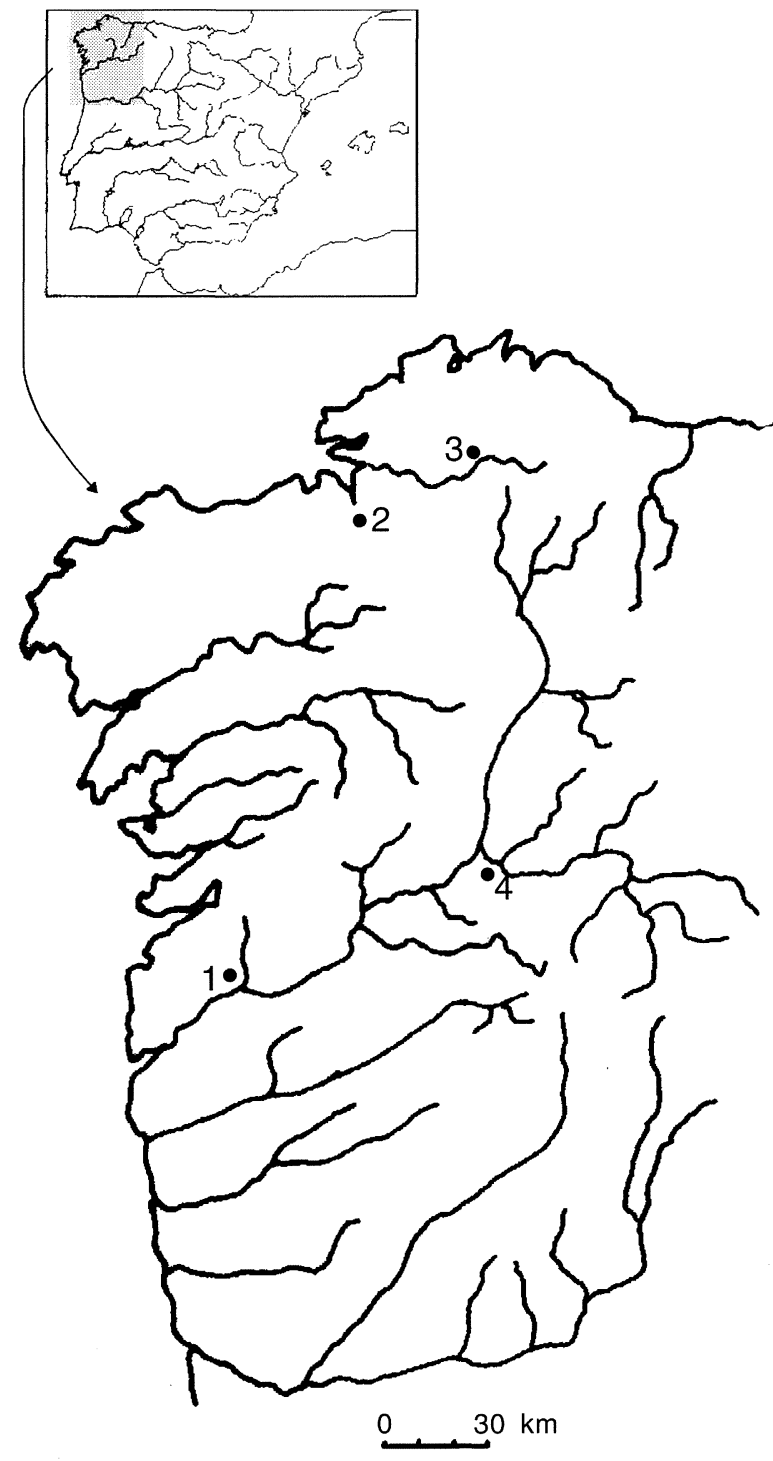

Fig. 1. Localización del área de estudio dentro de la Península Ibérica con indicación de los principales lugares citados en el texto. 1: Tui (Pontevedra). 2: Betanzos (A Coruña). 3: Santa María de As Pontes de García Rodríguez (Lugo). 4: San Estebo de Ribas de Sil (Ourense).

do, se especifica que "pertransit ad Mamula de Gutilanes" (Carneiro, 1995: 299).

Tenemos en esta cita la primera alusión conocida, dentro de la documentación gallega, a un monumento megalítico, entre otros restos prehistóricos que funcionan como mojones. Este uso podría ser, en cierta manera, heredero de una costumbre antigua. Así lo comentaremos en el próximo apartado. Pero lo que nos interesa comprobar ahora es que esta utilización no hará sino proliferar.
De este modo, si nos introducimos en las colecciones diplomáticas medievales y modernas, encontramos, por un lado, expresiones como ad illam mamunam in prono ad Campellos, ad archa antiqua, per illas mamuas de Sancta Marina, ad illa mamoa de inter Ardilleiros et Eldar, per mammuam furatam, per arcam que est in monte super Vilarino, per illum lumbum inter ambas antas, per petras fitas, ad illam mamolam terrenam de Castrilu, per illas mamonas de Foranas de Vilar, ad alia arca pitrinia... y un largo etcétera, que se emplean para delimitar un territorio concreto utilizando referentes megalíticos que debemos suponer serían conocidos por todos.

Y contamos también, por otro lado, con topónimos del tipo grangiam de Modorra, hereditatem quan dicunt Archas, Monte de Meda, ad montem qui dicitur Meda, per strata usque ubi dicentAntas, grangiam de Archas, in loco qui dicitur Arca, in loco qui vocatur Mamoelas..., en los que el túmulo se convierte ya en símbolo visible del lugar que ocupa, y pasa a darle nombre.

El número total de referencias a posibles monumentos megalíticos que pudimos encontrar en este grupo de fuentes es de 253. Su presencia es especialmente acusada en los expedientes de apeo que limitan todo tipo de fincas en los siglos centrales de la Edad Media (3). No podemos tener la certeza de que todas las referencias recogidas aludan a monumentos prehistóricos. Sin embargo, su abundancia permite confirmar que la utilización de megalitos como marcos de término sería un fenómeno bastante generalizado en la Galicia de este tiempo.

Por razones de espacio no podemos extendernos en ejemplificaciones, pero sí nos detendremos en el extracto de un documento. Lo hemos escogido porque, por la cantidad de posibles megalitos a los que hace referencia, parece venir a ratificar que el recurso a los megalitos de término no es algo casual sino, incluso, una solución buscada. El documento data de 1169, reinando Fernando II, cuando se acota por primera vez el territorio de la ciudad de Tui (Pontevedra). En en la demarcación se citan numerosos vestigios arqueológicos: “... ad mamulam de inter superatam et antam... et ad petram fictam que est in portela Fredenandi... et venitur in castrarum et

(3) Los expedientes de apeo son aquellos documentos que acreditan la realización de deslindes y demarcaciones de fincas y heredades, señalándolas y limitándolas con cotos y mojones conocidos, para aclarar los límites, evitar ocultaciones, confirmar propiedades... Aquí incluimos también los contratos agrarios foros, arrendamientos, ventas...- en los que se hace explicitación de los lindes. 
ad montem Oloye ubi fuit civitas antiquitus condita... vadit ad archam de Petrafita... et per archas de Fofi... et per illas archas antiquas Sancti Salvatori de Gandera" (Archivo de la Catedral de Tui, 4/ 4: 76; tomado de Ferreira, 1988).

Semeja, en conclusión, que por su antigüedad, visibilidad y solidez, los monumentos megalíticos se convierten en puntos de referencia aceptados convencionalmente para diferentes demarcaciones espaciales. La aceptación general de estos elementos como marcos queda también atestiguada por la reiteración de los mismos, en distintos expedientes, a lo largo del tiempo. De manera ilustrativa recogemos un ejemplo tomado de los Tumbos del Monasterio de San Estebo de Ribas de Sil (Ourense): el 12 de enero del año 921, Ordoño II concede al monasterio numerosas tierras que aparecen delimitadas por varios mojones, entre ellos: “... ad mamola de Villare... et per illa petra scripta que est inter $\mathrm{Fa}$ ramontaos et Eiratella... et inde per Petra Fita, et inde per medium montium que vocitant Meta" (Duro, 1977: documento 1).

Sucesivamente, en los siglos posteriores Alfonso IX, Fernando IV, Pedro I, Juan I, Juan II y sus sucesores confirmarán estos privilegios $\mathrm{y}$, para hacerlo, se remitirán a los mismos lindes (documentos $11,62,76,92$ y 112). De aquí podemos inferir que esos marcadores siguen existiendo, siguen siendo conocidos, y son aceptados como tales.

Si queremos acercarnos más al presente y seguir comprobando la utilización de megalitos como marcos de término debemos acudir, como ya indicamos, al Catastro de Ensenada, que nos trae ya a la segunda mitad del siglo XVIII. Partiendo de él encontramos, en la antigua provincia de Betanzos, 44 citas a construcciones megalíticas que funcionan como lindes o confrontaciones. Veamos una muestra de ello: “... de aquí buelbe hacia Levante al marco da Moura, de éste a la mámoa del Abrigadoiro, confrontando con la feligresía de San Paio de Paradela, y buelbe al Norte a la mámoa de Couso de Catarrán..." (San Miguel de Codesoso, $1036 \mathrm{v}$.)

“... de éste sigue agua arriva... hasta Piedra Chantada, de allíal marco de Mariña Leda, de allí a la Peña Cruzada, de allí al marco de lomuo de Ada... de éste a la modoña de Cotillón... sigue a otra Peña Cruzada, desde ésta a la mama furada... sigue a la referida Peña dos Mouros..." (Santa María de las Puentes de García Rodríguez, 3356 r.v.)

Además del indudable interés de las mámoas y modoñas, quizá también las peñas cruzadas po- drían referirse a dólmenes, como la mama furada a un túmulo violado y la piedra chantada a un menhir. Asimismo, por la común vinculación popular entre el personaje mítico y el vestigio prehistórico, tal vez el marco da Moura, el lomuo de Ada, y la Peña dos Mouros pudieran remitirnos a monumentos megalíticos.

A diferencia de lo que ocurría en siglos anteriores, en el Catastro estas referencias aparecen cuando se describe el contorno de las feligresías, pero no encontramos megalitos funcionando como mojones en los lindes de fincas o heredades menores. Este dato debe ser tenido en cuenta y más adelante trataremos de darle explicación, en el apartado 6.3. Pero antes prosigamos nuestro recorrido por la Historia de los megalitos.

Llegados al siglo XIX, para encontrar menciones a monumentos megalíticos como referentes territoriales tendremos que acudir a otro género de fuentes: los diccionarios de topónimos. Del Diccionario Nomenclator de Villarroel (1810) y del Diccionario Geográfico de Madoz (1845-50) hemos podido extraer, respectivamente, 34 y 56 nombres de lugar alusivos a megalitos, tales como lugar de Mámoa, lugar de Madorras, feligresía de Santiago de la Medorra, etc. La existencia de este tipo de topónimos permite hablar de la pervivencia del megalito como identificador de demarcación; ahora bien, este papel territorial es ya muy vago, toda vez que un topónimo puede ser una herencia del pasado y, en consecuencia, que un lugar se llameMámoa en el siglo XIX no indica ya que el túmulo siga allí $o$, al menos, que se necesite ese referente para precisar el espacio que se quiera indicar. También volveremos sobre esta idea en la posterior interpretación (apartado 6.3).

\section{CONTINUIDADES Y RUPTURAS}

Conocidos los datos, podemos proceder ahora a interpretarlos. Hemos comentado inicialmente (apartado 2) cómo los monumentos megalíticos prehistóricos ejercieron, en su momento de construcción y apogeo, una función significativa en la estructuración del paisaje, ya fuese como marcadores de territorios explotables, de espacios habitables, de lugares de tránsito... Hemos comprobado después, sobre el territorio gallego, que estos mismos megalitos funcionan como marcos de territorio e identificadores de espacios, de forma recurrente, en épocas ya plenamente históricas (apartados 3

T. P., 58, n. ${ }^{\circ} 1,2001$ 
y 4). Inevitablemente, esta doble constatación nos lleva a plantear un concepto: el de continuidad.

Es sabido que con el fenómeno megalítico se inicia la verdadera apropiación humana del entorno natural: es la primera culturalización del paisaje. Este proceso marca el inicio de una nueva época y se pone de manifiesto en la construcción de unos colosales monumentos que -aparte de otras funciones- marcan el espacio en que se ubican. Numerosos siglos después volvemos a encontrarnos esos monumentos marcando también el espacio. ¿Qué explicación cabe? Si partimos de que la cultura, en esencia, no es más que un modo de conjugar armónicamente las necesidades sociales con las realidades del mundo físico (Clark, 1980: 160), nos parece evidente que al campesino gallego le resulta más cómodo, más armónico, emplear como demarcador un elemento que ya existe en el paisaje y que ha tenido esa función, en lugar de construir uno para tal fin. Nos parece, por tanto, que podemos hablar de continuidad.

Evidentemente, la concordancia no debe llevarnos a extremos a la hora de plantear paralelismos entre distintas sociedades, culturas y paisajes. Sin ir más lejos, el campesino medieval no acomete la labor de decidir un lugar y emplazar allí un marco territorial, sino que se limita a reutilizar uno que ya existe. El megalito en el tiempo, además, ha pasado de ser una obra de hombres a convertirse en un “accidente geográfico" (Ferreira, 1988: 37) perfectamente asimilado ya por el entorno. Asimismo, en el Megalitismo teníamos un "paisaje monumental", mientras que después existe un "paisaje parcelado" o "dividido" (Criado, 1993). Si para la época megalítica hablábamos de "estructuración" del paisaje, ahora tenemos que referirnos a una "síntesis y adaptación" (López y Pereira, 1995: 40-41). Finalmente, para huir de una historia totalizadora debemos tener presente que cada sociedad construye y percibe sus propios conceptos de espacio y tiempo de forma singular (Shanks y Tilley, 1987: 117-185; Thomas, 1990; Santos et alii, 1997; Criado y Villoch, 1998). Todas estas divergencias están marcando rupturas entre la Galicia megalítica y la Galicia histórica, diferencias que no podemos pasar por alto. Pero si bien la perspectiva de la continuidad puede servir como base para definir diferencias que, obviamente, existen entre grupos humanos de distintas épocas, y que probablemente responden a necesidades mentales y sociales distintas (Criado, 1991b: 250), también es pertinente hacer a la inversa (Shanks y Tilley 1987: 185): partir de la especi- ficidad histórica y contextual para entender después la reproducción y la transformación de la sociedad.

Vuelve de este modo a sugerirse la idea de continuidad: por encima de las rupturas, reproducción de estructuras. En un trabajo reciente se ha marcado la ruptura que se iniciaría en la Edad del Hierro, por el paso de un espacio estructurado esencialmente por líneas de tránsito a un paisaje más estático, estructurado a partir de puntos fijos (Santos et alii, 1997; o Parcero et alii, 1998). Si tenemos en cuenta que los marcos empleados para esas distintas modalidades de organización espacial siguen siendo, en muchos casos, los mismos megalitos, se ratifica la idea de que la percepción de un mismo espacio varía en función del sustrato cultural. Pero también se pone de manifiesto la necesidad de atender a las permanencias.

Según los datos de que disponemos, el primero en insinuar esta continuidad en el empleo de los monumentos gallegos como marcos de territorio fue Manuel Murguía (Martinón-Torres, 2000b). Su mente lúcida fue capaz de alumbrar varias hipótesis que la arqueología tardaría muchas décadas en desarrollar. Él apreció del megalito que "después de limitar el campo sagrado, fijar los límites territoriales de la tribu y ser testigo de las asambleas nacionales [en su época de construcción], vésele servir de piedra terminal y en tal concepto ser santificado por el campesino [en épocas históricas]" (Murguía, 1888: 85). Sin embargo, parece que su hipótesis no tuvo eco. Nuestro planteamiento pretende ahora avanzar sobre esa línea. Pensamos que mientras se mantiene esa relativa armonía por la conservación y uso de los mismos demarcadores territoriales, en el fondo se está perpetuando cierta estructuración del espacio que se inició en el Megalitismo. En consecuencia, ante el paisaje actual, que "nos muestra su forma objetiva pero nos oculta siempre las razones profundas por las cuales ha llegado a ser como es" (López y Pereira, 1995: 44), empezamos a encontrar luz para la interpretación: detrás de todo está la inercia histórica, que suele tender a la conservación. En el momento de establecerse el modo de vida campesino se llevó a cabo, mediante los monumentos, una organización cultural del paisaje. Y mientras ha pervivido ese modo de vida, ha tendido a mantenerse también esa estructuración. Nos hallamos ante un indicio del "eterno campesino neolítico" que definió Carlos Alonso del Real (1969).

De aquí puede inferirse que la extraordinaria fragmentación de la propiedad rural gallega podría 
ser heredera de la que tuvo lugar en la época megalítica. No en vano, los monumentos megalíticos gallegos se caracterizan -como las fincas ruralespor un elevado número y un modesto tamaño (4).Y esa fragmentación del paisaje gallego precisamente comienza a borrarse por la concentración parcelaria, las roturaciones, diversas obras públicas..., es decir, cuando el modo de vida campesino, iniciado en el Neolítico, sucumbe ante la industrialización. Puede ser éste el momento de retomar la cita de ChristopherTilley (1993: 163-4) con la que iniciábamos este trabajo: mientras existe el mundo rural, el megalito sigue "funcionando". Sólo cuando la ciudad y su mundo acaban con el modo de vida rural tradicional, entonces se apaga el "aura" del megalito. Aquí ha tratado de sugerirse una explicación a este fenómeno.

Por cautela, no podemos dejar de insistir en que el significado de un paisaje estructurado no es inherente a él, aunque los marcadores puedan ser los mismos. Al contrario, su significado que depende de las ideas y convenciones de la sociedad que lo ocupa (Thomas, 1990: 169). Sin embargo, por lo que hemos visto, en un sentido práctico y material, por la perpetuación de los referentes que estructuran ese espacio, sí creemos que se pueden apreciar continuidades.

\section{PERSPECTIVAS EN LA INVESTIGACIÓN}

En el apartado anterior hemos presentado ya una propuesta interpretativa que trata de vincular los megalitos de término prehistóricos con los históricos. No obstante, en la realización de este trabajo nos hemos planteado otros interrogantes, y han surgido una serie de conclusiones que no queremos dejar de exponer.

\subsection{Las fuentes escritas y la prospección arqueológica}

Cualquier manual de metodología arqueológica señala, aun superficialmente, la utilidad de los viejos documentos, libros o mapas como fuentes para la localización de yacimientos. En ellos podemos encontrar referencias a restos arqueológicos cuya

(4) Se calcula que en Galicia habrá unos cinco mil túmulos, dispersos por todo el territorio, con una media de entre 15 y $30 \mathrm{~m}$ de diámetro (Rodríguez Casal, 1990, 1997). existencia puede haber desaparecido de la memoria colectiva. Para el Megalitismo, sin embargo -como en otros muchos ámbitos de investigación arqueológica-, la mayor parte de los trabajos de prospección se inician directamente sobre el terreno o, a lo sumo, revisando la literatura arqueológica anterior vertida sobre la zona que se quiere prospectar.

Llevado a cabo nuestro vaciado de documentos medievales y modernos, y ante la cantidad de citas a megalitos que pudimos encontrar, nos vemos en situación de valorar en su justa medida el registro de fuentes antiguas como primer paso para la localización de monumentos. Por las limitaciones de espacio no podemos presentar aquí la base de datos confeccionada a partir de todas esas citas, pero sí debemos advertir, al menos, que esas referencias documentales aportan precisiones de interés para localizar monumentos megalíticos (cfr. MartinónTorres, e.p.a). Si los antiguos gallegos conocían tal o cuạl demarcación por tener al lado un túmulo, nosotros podemos hoy realizar el proceso inverso: tomar otros referentes conocidos para encontrar esa finca y, a partir de ella, el megalítico marco al lado del cual se hallaba.

Del mismo modo, recoger los topónimos que aparecen en documentos y libros antiguos se manifiesta como otra metodología de prospección útil, toda vez que algunos topónimos han desaparecido en la actualidad, y no por ello deberían dejar de tenerse en cuenta. Valga como ejemplo el caso del Monte das Mámoas, citado de esta manera en el Catastro de Ensenada, pero que hoy ha mudado su denominación por la de Monte das Moas: en este monte se han podido catalogar doce monumentos megalíticos (5).

\subsection{Las fuentes escritas y los estudios de emplazamiento}

Continuando en la línea de lo anterior, a partir de las referencias antiguas podemos localizar megalitos en el campo pero también, posiblemente, comprobar que ya no existen en el lugar que se indicaba. En este caso, lo que podría parecer una pista fracasada para un trabajo de prospección orientado sólo al catálogo y a la conservación, se convierte en

(5) Cfr. J.A. Fernández Malde: Contexto ambiental e implicaçons socioculturais do Megalitismo das bacias do Mendo e Mandeo (comarca de Betanços). Trabajo de Investigación de Tercer Ciclo inédito. Universidad de Santiago de Compostela, 1999. 
un dato de especial relevancia para aquél que quiera realizar un análisis completo del emplazamiento de los megalitos.

Así, cuando pretendemos reconstruir la configuración del paisaje de la época megalítica no podemos partir únicamente de los monumentos que persisten en el paisaje contemporáneo: los datos estarán desvirtuados. Por ejemplo, estudios de interés como los que tratan de poner en relación la distribución de los túmulos gallegos con el tradicional reparto del espacio rural entre campos de cultivo -intensivo y extensivo- y campos incultos (Criado et alii, 1986; Criado, 1988; Vaquero, 1991-2; Criado y Vaquero, 1993: 227-236) toman normalmente sus datos de prospecciones sobre el terreno. Creemos que sus datos podrían conjugarse con aquéllos que aporta la revisión de fuentes escritas. El hecho de que la mayor parte de las referencias documentales aludan sobre todo a megalitos emplazados en zonas bajas, mientras que hoy encontramos los monumentos, fundamentalmente, en zonas topográficas medias y altas, nos inclina a esa consideración. Un estudio realizado entre el archivo y el campo podría abrirnos vías de análisis nuevas o, cuando menos, más completas.

\subsection{La valoración de los megalitos en la Historia: una interpretación}

En la Introducción a este trabajo (apartado 1) apuntábamos tres potenciales del monumento megalítico, tres funciones del mismo que se han ido valorando con intensidad variable a lo largo de los siglos: un papel social-territorial, un papel simbólico-mítico, y un papel histórico-arqueológico. Aquí nos hemos centrado en el primero de esos aspectos, para ver cómo los hombres fueron aprovechando esta utilidad de los monumentos, hasta que su uso decayó. A continuación trataremos de explicar la particular evolución de esa consideración social-territorial del megalito, relacionándola con los otros dos valores definidos y con la destrucción histórica de monumentos por motivos variables. Para ello partimos de que esos tres valores o potenciales pueden haber funcionado en la Historia como impulsos antagónicos, inversamente proporcionales.

Hemos comprobado la constante utilización de megalitos como marcos de término, especialmente acusada en los siglos centrales de la Edad Media. Teniendo presente la idea de continuidad y armonía con el pasado que expusimos antes, entendimos que el campesino medieval empleaba como demarcadores unos mojones -los megalitos- que ya estructuraban el paisaje en el pasado. En este período, por tanto, la valoración social-territorial del monumento sería la predominante, aunque éste también tuviese un valor mítico. En consecuencia, pensamos que un estudio del patrón de conservación-destrucción de los monumentos megalíticos podría ponerse en relación con ese pretérito empleo de los mismos como marcos de territorio: por su propia comodidad, un campesino trataría de evitar la destrucción de un megalito de término que, en última instancia, funciona como un cartel que indica hasta dónde llega su propiedad. En cambio, no se opondría a la destrucción de otros que, por carecer de esa utilidad práctica, no constituirían sino estorbos.

Sin embargo, avanzando en el tiempo llegamos al siglo XVII y encontramos una realidad muy distinta, que se pone de manifiesto en el famoso proceso judicial dẹ Vázquez de Orjas (6). En esta épo$\mathrm{ca}$, los paisanos gallegos se lanzan en masa a la destrucción de túmulos, pasando por alto su utilidad práctica o territorial, y valorando, por el contrario, el potencial de los megalitos como posibles escondrijos de tesoros. Tenemos, de este modo, que la consideración del monumento como elemento simbólico-mítico, que habría existido siempre, aparece ahora como la más importante, y condiciona un nuevo patrón de conservación-destrucción. Nuestra hipótesis se asienta sobre la idea de que, si se potencia tanto en esta época el valor mítico de los túmulos, esto viene favorecido porque, a estas alturas, la función social-territorial de la mayor parte de ellos es ya prescindible: nuevos recursos técnicos facilitan el cercado de las fincas con muros. Para estos muros se aprovecharán muchas veces, precisamente, las grandes piedras de los megalitos. Por eso puede liberarse el instinto mítico, y en esta época se habrían destruido gran parte de los monumentos que encontrábamos en los expedientes de apeo medievales y que, por el contrario, difícilmente aparecerán en una prospección convencional.

(6) Actualmente preparamos la publicación de los folios procesales del pleito que a principios del siglo XVII protagonizó el licenciado Vázquez de Orjas, por la destrucción furtiva de cientos de mámoas gallegas en busca de tesoros que supuestamente pertenecían a la Hacienda Real. A través de esta documentación se aprecia claramente, entre otros aspectos, la intensa valoración simbólico-mítica de los megalitos imperante en este tiempo. Los resultados preliminares de nuestro trabajo pueden verse en Martinón-Torres, e.p.b, o, más brevemente, en Martinón-Torres y Rodríguez Casal, 2000. 
Continuando con nuestra línea argumental, comprobamos esa progresiva pérdida del interés socialterritorial del megalito en el siglo XVIII, a través del Catastro de Ensenada. En él encontramos los megalitos de término tan sólo en los lindes de las feligresías, ya no en todas las propiedades agrarias: los monumentos que conservan ese uso son únicamente los emplazados en zonas cercanas a las divisorias, más apartados de las áreas que los gallegos suelen cultivar, conocer y registrar en busca de míticos tesoros. Finalmente, el valor territorial del megalito será ya mínimo en el siglo XIX, cuando sólo persiste, como resquicio del pasado, en la toponimia.

Tratemos de plantear esta argumentación en otros términos. Para ello puede ser útil la perspectiva adoptada por Felipe Criado (1989a: 86 ss.) para explicar las sucesivas etapas del fenómeno megalítico. Según su análisis, los diferentes estadios por los que pasó el Megalitismo vienen dados por los distintos modos en que se fue resolviendo la tensión entre dos valores: monumentalidad externa y monumentalidad interna. Nuestra propuesta es que la idea de tensión entre los valores interno y externo 'del monumento puede ser útil también para comprender la valoración de los megalitos que se dará después. Así, en la Edad Media priman los aspectos espaciales, la monumentalidad externa, por el empleo de los megalitos como marcos de territorio. En la época de Vázquez de Orjas (siglo XVII), en cambio, se centra la atención sobre la monumentalidad interna, el tesoro escondido en el túmulo.

En esta secuencia falta el tercer valor, el histórico-arqueológico. Para entender su aparición debemos recurrir, de nuevo, a la idea de tensión y oposición entre las distintas consideraciones de los monumentos. Así, en Galicia, el megalito no será observado como fuente de información sobre el pasado, como objeto arqueológico, mientras no se sublimen los valores social-territorial y simbólicomítico. Aunque en tiempos de Vázquez de Orjas todos los gallegos debieron enterarse de que su país estaba sembrado de tumbas de antepasados gentiles, los túmulos no aparecen en los libros de Historia. Creemos que esto se debe a que el megalito sigue plenamente integrado en el ámbito de lo mítico. Sólo en el siglo XVIII, a partir de la Ilustración, cuando se combatan sistemáticamente las creencias míticas del folklore popular, y cuando el papel demarcador del megalitos se haya reducido ostensiblemente, podrá atenderse a su dimensión histórico-arqueológica. Los primeros historiadores gallegos que se ocupen del Megalitismo serán pre- cisamente personajes que, por su particular situación socioeconómica y cultural, no precisan del monumento como marco de término ni creen los fabulosos mitos (cfr. Martinón-Torres, 2000a).

Si se nos permite la osadía de un paralelismo nietzscheano, diremos que la dimensión territorial representa la pulsión apolínea, mientras que lo simbólico-mítico sería la fuerza dionisíaca. Y que, como la tragedia ática, el Megalitismo entra en la escena de la erudición histórica cuando se sintetiza, al tiempo que se sublima, esa doble tensión.

\subsection{De la revisión historiográfica a la crónica}

El artículo que aquí concluye parte de la revisión de lo escrito en el pasado sobre un objeto de estudio que hoy seguimos tratando de conocer. Sin embargo, nuestra pretensión no ha sido la de llevar a cabo una revisión historiográfica, entendida como una antọlogía simple y lineal de fuentes. Hemos tomado una variable, el valor social-territorial del monumento megalítico, para tratar de reconstruir cómo se aprovechó esta funcionalidad a lo largo de los siglos. A partir de esta reconstrucción intentamos presentar planteamientos o perspectivas que pudieran ser útiles para trabajos posteriores. Finalmente, desde una perspectiva más amplia, tratamos de apuntar cómo habría evolucionado en el tiempo la consideración otorgada por los hombres a los megalitos, y de sugerir una interpretación a este proceso.

Nuestra condición de autor nos impide valorar hasta qué punto son acertadas nuestras conclusiones y correcta la exposición de las mismas. Sin embargo, no nos gustaría terminar sin plantear una idea que está en el fondo de este trabajo. Los constructores de megalitos desaparecieron hace miles de años. Sin embargo, muchos monumentos megalíticos permanecieron en el paisaje, y por eso pudieron ser objeto de nuevos usos y de nuevas reflexiones, que llegan hasta hoy. Extinguidas la sociedad y la cultura que dieron origen a los megalitos, nuevas sociedades y nuevas culturas fueron, sucesivamente, otorgándoles nuevos significados y nuevas funciones. El monumento ha seguido viviendo y, por tanto, consideramos pertinente estudiar y comprender su vida entera, no sólo su nacimiento.

Este planteamiento no es inédito, sino que aparece por primera vez, según nuestros datos, en un trabajo de Glyn Daniel (1972). Posteriormente, en los últimos años han surgido diversos artículos que se articulan sobre esa base teórica o que, de algún 
modo, la reformulan (Bradley, 1984, 1993; Caamaño y Criado, 1991-2; Chippindale, 1994; Hingley, 1996; Patton, 1996; Holtorf, 1997, 1998a, b; Blake, 1998; Newman, 1998; Semple, 1998; Williams, 1998), varios de ellos recogidos en un libro dedicado precisamente a la reutilización de monumentos antiguos (Bradley y Williams, 1998). Cuando se empezaba a estimular la posibilidad de estudiar los megalitos diacrónicamente se escribió que "sería esta una historia curiosa y difusa, difícil de historiar y recomponer, una historia que se escabulle entre las grietas de una especialización profesional que hace de todos nosotros esmerados portavoces de períodos concretos y nos cierra el acceso a esa historia sin períodos específicos" (Caamaño y Criado, 1991-2: 47). Desde este punto de vista, reconstruir esta Historia se presenta para nosotros como un reto, un desafío que debemos aceptar. Acercarnos a la Historia de los megalitos nos abre una puerta para comprender paisaje y cultura. Es una Historia de la que son partícipes los constructores de megalitos, pero también los campesinos, las mouras encantadas, los buscadores de tesoros, los paseantes ilustrados, los canteros devastadores, los propios prehistoriadores... todos los que, a lo largo de la vida de los monumentos, hemos tenido relación con ellos. Se nos plantea así el objetivo de reconstruir una crónica del monumento megalítico en las distintas sociedades y tiempos.

Un estudio paradigmático en este sentido es el que realizó Cornelius J. Holtorf (1998a, b) sobre las "biografías" de los megalitos de Mecklenburg-Vorpommern. En él se reconstruye ejemplarmente la vida secular de los monumentos como depositarios de memorias colectivas ancestrales y acumulativas. Con nuestro trabajo pretendíamos, en última instancia, aproximarnos a este planteamiento pero, a diferencia del anterior, considerando los megalitos más como land-marks que como time-marks, y partiendo de que en las fuentes escritas antiguas, aun indirectamente, se puede encontrar información para esta labor. Ambas perspectivas quizá pudieran complementarse. Si de alguna manera hemos sido capaces de dar un paso en ese camino, si hemos aportado algo a la escritura de esa crónica, entonces nos damos por satisfechos.

\section{AGRADECIMIENTOS}

Antón A. Rodríguez Casal me introdujo en las fuentes de la Historia con perspectiva de prehisto- riador y dirigió mi Tesis de Licenciatura. Sólo entonces surgió la idea inicial de este trabajo. M. ${ }^{a}$ Isabel Martínez Navarrete me animó a escribir este artículo y después no se cansó de aconsejarme. María R. Serna me sugirió útil bibliografía sobre el megalitismo de la Cornisa Cantábrica.A todos ellos manifiesto mi más sincero agradecimiento. Evidentemente, todos los errores e imprecisiones son únicamente responsabilidad del que firma.

El trabajo fue escrito en 1999 en la Universidad de Santiago de Compostela mientras disfrutaba una beca de la Secretaría Xeral de Investigación e Desenvolvemento de la Xunta de Galicia, y completado después en el Instituto de Arqueología de Londres mientras disfrutaba una beca otorgada por la Fundación Pedro Barrié de la Maza y The British Council. También a estas instituciones expreso mi gratitud.

\section{BIBLIOGRAFÍA}

Alonso del Real y Ramos, C. (1969): "Las raíces de Galicia". Grial, 24: 163-172.

Amor MeILÁn, M. (1918): Historia de la provincia de Lugo, vol. I: Edad Prehistórica. Excma. Diputación Provincial de Lugo. Lugo.

ANDRÉs RupÉREZ, M. ${ }^{a}$ T. (1999): “Los caminos y los sepulcros megalíticos”. En M. ${ }^{\mathrm{a} A}$. Magallón (coord.): $\mathrm{Ca}$ minos y comunicaciones en Aragón. Institución "Fernando el Católico" (C.S.I.C.) - Excma. Diputación Provincial de Zaragoza. Zaragoza: 29-41.

Barros Silvelo, R. (1875): Antigüedades de Galicia. Imprenta de D. Domingo Puga. A Coruña.

Beguiristáin Gúrpide, M. ${ }^{a}$ A. y Vélaz Ciaurriz, D. (1999): "Megalitos, paisaje y memoria. Un estado de la cuestión". Memoria y civilización, 2: 317-327.

BlaKe, E. (1998): "Sardinia's nuraghi: four millenia of becoming”. World Archaeology, 30 (1): 59-71.

BRADLEY, R. (1984): "Studying monuments". En R. Bradley y J. Gardiner (eds.): Neolithic Studies: A Review of Some Current Research. British Archaeological Reports, British Series 133. Oxford: 61-66.

- (1993): Altering the Earth.The Origins of Monuments in Britain and Continental Europe. Society of Antiquaries of Scotland. Edinburgh.

Bradley, R. y Williams, H. (eds.) (1998): "The Past in the Past: The Reuse of Ancient Monuments". World Archaeology, 30 (1).

Caamaño Gesto, J.M. y Criado Boado, F. (1991-92): “La medorra de Fanegas (Sobrado dos Monxes, A Coruña). Un monumento megalítico reutilizado en época romana". Brigantium, 7: 7-89.

Carneiro Rey, J.A. (1995): “El fenómeno tumular en Na-

T. P., 58, n. ${ }^{\circ} 1,2001$ 
rón: análisis de localización". Estudios Mindonienses, 11: 293-362.

CASTILlo López, A. del (1927): "Hachas de bronce de talón (Hallazgo de un depósito)". Boletín de la RealAcademia Gallega, 17, 194: 33-42.

Chippindale, C. (1994): Stonehenge Complete. Thames and Hudson. London.

Clark, G. (1980): Arqueología y sociedad (Reconstruyendo el pasado histórico). Akal. Madrid (1. a ed. 1939).

CosTA, P.A. de J. da (1965): Liber Fidei Sanctae Bracarensis Ecclesiae. Ediçao crítica, I. Assembleia Distrital de Braga. Braga.

- (1978): Liber Fidei Sanctae Braccareinsis Ecclesiae. Edição crítica, II. Assembleia Distrital de Braga. Braga.

Criado Boado, F. (1988): "Mámoas y rozas: panorámica general sobre la distribución de los tumulos megalíticos gallegos". Actas do Colóquio de Arqueologia do Noroeste Peninsular (Porto-Baiâo, 22-24 setembro 1988), I. Trabalhos de Antropologia e Etnologia, 28: 151-169.

- (1989a): "Megalitos, espacio, pensamiento". Trabajos de Prehistoria, 46: 75-98.

- (1991a): "Tiempos megalíticos y espacios modernos". Historia y crítica, 1: 85-108.

- (dir.) (1991b): Arqueología del Paisaje. El área Bocelo-Furelos entre los tiempos paleolíticos y medievales. Xunta de Galicia, Consellería de Cultura e Xuventude, Dirección Xeral do Patrimonio Histórico e Documental. Santiago de Compostela.

- (1993): "Límites y posibilidades de laArqueología del Paisaje”. Spal, 2: 9-55.

Criado Boado, F.; Aira Rodríguez, M. aj. y Díaz-Fierros ViQUEIRA, F. (1986): La construcción del paisaje: Megalitismo y ecología en la sierra de Barbanza (Galicia). Arqueoloxía/Investigación, 6. Xunta de Galicia, Dirección Xeral de Patrimonio Artístico e Monumental. Santiago de Compostela.

Criado Boado, F. y Grajal, M. (1981): "Relación entre la distribución de mámoas y el medio físico en la zona de Sobrado-Curtis". Brigantium, 2: 7-26.

Criado Boado, F. y Vaquero Lastres, J. (1993): "Monumentos, nudos en el pañuelo. Megalitos, nudos en el espacio. Análisis del emplazamiento de los monumentos tumulares gallegos". Espacio, Tiempo y Forma. Prehistoria y Arqueología, 6: 205-248.

Criado Boado, F. y Villoch VÁzquez, V. (1998): "La monumentalización del paisaje: percepción actual y sentido original en el megalitismo de la sierra de Barbanza (Galicia)". Trabajos de Prehistoria, 55 (1): 63-80.

DANIEL, G. (1972): Megaliths in History. Walter Neurath Memorial Lecture. Thames and Hudson. London.

Delibes de Castro, G.; Benet Jordana, N.; Pérez Martín, R. y Zapatero Magdaleno, P. (1997): "De la tumba dolménica como referente territorial al poblado estable: notas sobre el hábitat y las formas de vida de las comunidades megalíticas de la Submeseta norte". En A.A.
Rodríguez Casal (ed.): O NeolíticoAtlántico e as orixes do Megalitismo. Actas del Coloquio Internacional (Santiago de Compostela, 1996). Santiago de Compostela: $779-808$.

Díez Castillo, A.; Díaz Casado, Y. y Robles Fernández, G. (1995): "La neolitización en las comarcas de Liébana y Polaciones (Cantabria): implicaciones socio-económicas". EnV. Oliveira Jorge (coord.): I Congresso de Arqueologia Peninsular (Porto, 1993). Actas, VI. Trabalhos de Antropologia e Etnologia, 35 (2): 55-71.

Díez Sanjurjo, M. (1904): "Los caminos antiguos y el itinerario n. ${ }^{\circ} 18$ de Antonino en la provincia de Orense". Boletín de la Comisión Provincial de Monumentos Históricos y Artísticos de Orense, 2, 37: 221-228.

Duro Peña, E. (1977): El monasterio de San Esteban de Ribas de Sil. Instituto de Estudios Orensanos "Padre Feijoó" de la Diputación Provincial. Ourense.

Eguileta Franco, J.M. (1997): "Megalitos y coordenadas espaciales en la Baixa Limia gallega: ¿una neolitización tardía en la Galicia interior?". EnA.A. Rodríguez Casal (ed.): O Neolítico Atlántico e as orixes do Megalitismo. Actas del Coloquio Internacional (Santiago de Compostela, 1996). Santiago de Compostela: 553-571.

- (1999): A Baixa Limia galega na Prehistoria Recente. Arqueoloxía dunha paisaxe na Galicia interior. Excma. Diputación Provincial de Ourense. Ourense.

Ferreira Priegue, E. (1988): Los caminos medievales de Galicia. Anexo 9 del BoletínAuriense. MuseoArqueolóxico Provincial de Ourense. Ourense.

Ferro Couselo, J. (1952): Los petroglifos de término y las insculturas rupestres de Galicia. Talleres gráficos de Miguel López Elizalde. Ourense.

Filgueira Valverde, J. y García Alén, A. (1977): “Inventario de monumentos megalíticos en la provincia de Pontevedra". El Museo de Pontevedra, 31: 49-130.

FLEMING, A. (1973): "Tombs for the Living". MAN, 8: 177193.

Galán Domingo, E. y Martín Bravo, A.M. ${ }^{a}$ (1991-92): "Megalitismo y zonas de paso en la cuenca extremeña del Tajo". Zephyrus, 44-45: 193-205.

Hingley, R. (1996): "Ancestors and identity in the later prehistory of Atlantic Scotland: the reuse and reinvention of Neolithic monuments and material culture". World Archaeology, 28 (2): 231-243.

Holtorf, C.J. (1997): "Beyond Chronographies of Megaliths: Understanding Monumental Time and Cultural Memory". En A. A. Rodríguez Casal (ed.): O Neolítico Atlántico e as orixes do Megalitismo. Actas del Coloquio Internacional (Santiago de Compostela, 1996). Santiago de Compostela: 101-114.

- (1998a): "The life-histories of megaliths in Mecklenburgh-Vorpommern (Germany)". World Archaeology, 30 (1): 23-38.

- (1998b): Monumental Past. The Life-histories of Megalithic Monuments in Mecklenburgh-Vorpommern (Germany). Monografía en formato hipertexto y en

T. P., 58, n. ${ }^{\circ} 1,2001$ 
permanente reactualización. [http://citd.scar.utoronto. ca/citdpress/holtorf/0.1.html]

López Paz, P. y Pereira Menaut, G. (1995): "La tierra y los hombres: paisaje político, paisaje histórico". Studia Historica. Historia Antigua, 13-14: 39-60.

Maciñeira Y PARdo de LAMA, F. (1942): “Túmulos prehistóricos. Inventario descriptivo de los doscientos ochenta y seis túmulos prehistóricos hasta ahora descubiertos en la avanzada comarca del Cabo Ortegal". Boletín de la Real Academia Gallega, 23, 266: 21.

- (1947): Bares. Puerto hispánico de la primitiva navegación occidental. Edición de F. Bouza Brey. Instituto Padre Sarmiento de Estudios Gallegos - C.S.I.C. Santiago de Compostela.

MAdOz, P. (1845-50): Diccionario Geográfico-EstadísticoHistórico de España y sus posesiones de Ultramar. Galicia. Ed. facsímil a cargo de Juan J. Vega González. Libros Galicia. Santiago de Compostela, 6 vols.

MARTinón-Torres, M. (2000a): "Análisis del 'Megalitismo Céltico' en la Galicia del siglo XIX”. Gallaecia, 19: 287-309.

- (2000b): "Murguía e a arqueoloxía galega". Boletín da Real Academia Galega, 361: 221-244.

- (e.p.a): Os monumentos megalíticos despois do Megalitismo. Prospección e Historia dos megalitos galegos a través das fontes escritas ( $s$. VI - s. XIX). (V Premio de Investigación Xesús Ferro Couselo) (Concello de Valga). Valga (Pontevedra).

- (e.p.b): "El Padre Sarmiento y el descubrimiento del Megalitismo gallego como fenómeno de interés arqueológico". Cuadernos de Estudios Gallegos, 112.

Martinón-Torres, M. y Rodríguez Casal, A.A. (2000): "Aspectos historiográficos del Megalitismo gallego: de la documentación medieval al siglo XIX". En Actas do III Congresso de Arqueologia Peninsular (Vila Real, Portugal, 1999), III Neolitização e Megalitismo da Península Ibérica. ADECAP. Porto (Portugal): 303319.

Monteagudo, L. (1954): “Nombres de túmulos y dólmenes en Galicia y Portugal". Revista de Filología Española, 38: $100-115$.

Murguía, M. (1888): Galicia. Col. España. Sus monumentos y artes. Su naturaleza é Historia. Establecimiento Tipográfico - Editorial de Daniel Cortezo y C. ${ }^{a}$. Barcelona.

Newman, C. (1998): "Reflections on the making of a 'royal site' in early Ireland". World Archaeology, 30 (1): 109-126.

Parcero Oubiña, C.; Criado Boado, F. y Santos Estévez, M. (1998): "Rewriting landscape: incorporating sacred landscapes into cultural traditions". WorldArchaeology, 30 (1): 159-176.

Patton, M. (1996): "La Hougue Bie à Jersey: transformation d'un monument du Néolithiue à nos jours". Bulletin de la Société Préhistorique Française, 93: 298-300.
Pena Graña, A. (1991): Narón, un concello con historia de seu. Servicio Sociopedagóxico Municipal. Narón.

Ramil Rego, E. (1997): "Megalitismo en el Concello de Vilalba (Lugo): su relación con el medio natural". En A.A. Rodríguez Casal (ed.): O NeolíticoAtlántico e as orixes do Megalitismo. Actas del Coloquio Internacional (Santiago de Compostela, 1996). Santiago de Compostela: $537-552$.

RENFREw, C. (1975): Before Civilization. The Radiocarbon Revolution and Prehistoric Europe. Jonathan Cape. London (1. ${ }^{\text {a }}$ ed. 1973).

- (1976): "Megaliths, Territories and Populations". En S.J. de Laet (ed.): Acculturation and Continuity in Atlantic Europe. Dissertationes Archaeologicae Gandenses, XVI. De Tempel. Brugge: 298-320.

- (1983): "The Megalithic Builders of Western Europe". En C. Renfrew (ed.): The Megalithic Monuments of Western Europe. Thames and Hudson. Londres: 8-17.

Rodríguez CASAl, A.A. (1990): O Megalitismo. A primeira arquitectura monumental de Galicia. Servicio de Publicacións e Intercambio Científico da Universidade de Santiago de Compostela. Santiago de Compostela.

- (1997): "Neolítico e Megalitismo en Galicia”. EnA.A. Rodríguez Casal (ed.): O NeolíticoAtlántico e as orixes do Megalitismo. Actas del Coloquio Internacional (Santiago de Compostela, 1996). Santiago de Compostela: 447-462.

Rodríguez GonzÁlez, X. (1996): "Don Xesús Ferro Couselo: investigación arqueolóxica". En Xesús Ferro Couselo: lembranzas, actividade, inquedanza. Anexo 21 del Boletín Auriense. Ourense: 179-189.

Rojo Guerra, M.A. (1994): "La relación hombre/espacio en el horizonte megalítico de La Lora". En V. Oliveira Jorge (coord.): I Congresso de Arqueologia Peninsular (Porto, 1993). Actas, IV. Trabalhos de Antropologiae Etnologia, 34 (3-4): 81-98.

Santos Estévez, M.; Parcero Oubiña, C. y Criado Boado, F. (1997): "De la arqueología simbólica del paisaje a la arqueología de los paisajes sagrados". Trabajos de Prehistoria, 54 (2): 61-80.

Sarmiento, M. (1850):"Manuscrito". Recogido por L. Martínez de Padín (1850): Historia política, religiosa $y$ descriptiva de Galicia, 2. Establecimiento tipográfico de A. Vicente. Madrid: 73-75.

SEMPLE, S. (1998): "A fear of the past: the place of the prehistoric burial mound in the ideology of middle and later Anglo-Saxon England". World Archaeology, 30 (1): 109-126.

Shanks, M. y Tilley, C. (1987): Social Theory and Archaeology. Cambridge University Press. Cambridge.

Shee Twohig, E. (1997): "Perspectives on the Megaliths of North West Europe". EnA.A. Rodríguez Casal (ed.): $O$ Neolítico Atlántico e as orixes do Megalitismo. Actas del Coloquio Internacional (Santiago de Compostela, 1996). Santiago de Compostela: 117-127. 
SHERRATT,A. (1990): “The genesis of megaliths: monumentality, ethnicity and social complexity in Neolithic north-west Europe". WorldArchaeology, 20 (2): 147-167.

TEIRA MAYOLINI, L.C, (1994): El megalitismo en Cantabria. Aproximación a una realidad arqueológica olvidada. Servicio de Publicaciones de la Universidad de Cantabria. Santander.

Tilley, C. (1993): "Art, Architecture, Landscape [Neolithic Sweden]". Historia y crítica, 3: 163-178.

- (1994): A phenomenology of landscape. Places, paths and monuments. Explorations in Anthropology. Berg. Oxford/Providence.

- (1996): "The power of rocks: topography and monument construction on Bodmin Moor". WorldArchaeo$\log y, 28$ (2): 161-176.

Thомаs, J.S. (1990): "Monuments from the inside: the case of the Irish megalithic tombs". WorldArchaeology, 22 (2): 168-178
TRIGGER, B.G. (1992): Historia del pensamiento arqueológico. Crítica. Barcelona.

VAQUeRo LASTRES, J. (1991-92): "Del análisis del emplazamiento al estudio de la distribución de túmulos en el NW". Brigantium, 7: 151-176.

VILlarroel, J. de (1810): Diccionario Nomenclator de las ciudades, villas, aldeas, caserías, cotos, ventas, castillos, y prioratos de todo el Reyno de Galicia. Imprenta de D. Juan Francisco Montero. Santiago de Compostela.

VilLOCH, V. (1995): "Monumentos y petroglifos: la construcción del espacio en las sociedades constructoras de túmulos del noroeste peninsular". Trabajos de Prehistoria, 52 (1): 39-55.

WiLliams, H. (1998): "Monuments and the past in early Anglo-Saxon England". World Archaeology, 30 (1): 90-108. 